\title{
Sub-MeV Bosonic Dark Matter, Misalignment Mechanism and Galactic Dark Matter Halo Luminosities
}

\author{
Qiaoli Yang $^{1 *}$ and Haoran $\mathrm{Di}^{2 \dagger}$ \\ ${ }^{1}$ Department of Physics, Jinan University, Guangzhou 510632, China \\ ${ }^{2}$ School of Physics, Huazhong University of Science and Technology, Wuhan 430074, China
}

\begin{abstract}
We explore a scenario that dark matter is a boson condensate created by the misalignment mechanism, in which a spin 0 boson (an axion-like particle) and a spin 1 boson (the dark photon) are considered, respectively. We find that although the sub-MeV dark matter boson is extremely stable, the huge number of dark matter particles in a galaxy halo makes the decaying signal detectable. A galaxy halo is a large structure bounded by gravity with a typical $\sim 10^{12}$ solar mass, and the majority of its components are made of dark matter. For the axion-like particle case, it decays via $\phi \rightarrow \gamma \gamma$, therefore the photon spectrum is monochromatic. For the dark photon case, it is a three body decay $A^{\prime} \rightarrow \gamma \gamma \gamma$. However, we find that the photon spectrum is heavily peaked at $M / 2$ and thus can facilitate observation. We also suggest a physical explanation for the three body decay spectrum by comparing the physics in the decay of orthopositronium. In addition, for both cases, the decaying photon flux can be measured for some regions of parameter space using current technologies.
\end{abstract}

Introduction: Mounting evidence shows that dark matter constitutes about one third of the total energy density or more than $80 \%$ of the matter density of our universe. In galaxy halos, the mass fractions of dark matter are even more dominated which can be as high as 95\% [17]. Although the existence of dark matter is widely accepted, the exactly nature of dark matter particles are still little known [8] besides that they are non-baryonic, weakly interacting and stable. There are many possible dark matter particle candidates, and two of them, i.e., the axion-like particles $\phi$ 9 21] and the hidden massive $\mathrm{U}(1)$ vector boson $A_{\mu}^{\prime}$ [22 30], are especially interesting because both can be produced from the misalignment mechanism [9 12, 14, 31, 35]. The misalignment mechanism does not require any particular interactions between the dark matter particles and the Standard Model sector as far as dark matter particles are bosons and massive in the very early universe. Therefore, the spin 0 case can be the axion or axion-like particle, in which case one of the "string axions" accounts for the majority of the dark matter, and the spin 1 case can be the dark photon.

If the dark matter is composed of axion-like particles or dark photons, then the dark matter could be a boson condensate [36] with a high number density. As these cosmic dark matter particles are not completely stable, they can decay into three (from the dark photon) or two (from the axion-like particle) photons. Certainly, to be a proper dark matter candidate the lifetime of the particle should be beyond the age of the universe, however, the luminosity of decaying photons from a dark matter halo can be measured due to the huge number of particles in a halo. In addition, we find that the signal of the decayed photons is very unique. For the axion-like particle case, it is a two body decay $\phi \rightarrow 2 \gamma$, so the energy spectrum

\footnotetext{
*qiaoli_yang@hotmail.com

†haoran_di@yahoo.com
}

of the photons is monochromatic at $E=M_{\phi} / 2$, where $M_{\phi}$ is the mass of the axion-like particle. For the dark photon case, although it is a three body decay, the energy spectrum is heavily peaked (see Fig.2) according to our calculation. The physical reason for this three body decay spectrum is understandable if we compare it with the spectrum of the orthopositronium decay [37], which we discuss in the following section.

Dark Matter Models and Misalignment Mechanism: Hypothetical particles in the hidden sector are generally interacting very weakly with the standard model sector. The hidden $\mathrm{U}(1)$ vector boson was originally proposed to be a massless particle that carried a long range force on dark matter. It was later realized that the massive vector bosons themselves could be a very good candidate for dark matter. Here, let us use $A_{\mu}^{\prime}$ to denote the hidden sector $\mathrm{U}(1)$ vector field. The Lagrangian is:

$$
\begin{aligned}
\mathcal{L} & =-\frac{1}{4}\left(F^{\mu \nu} F_{\mu \nu}+F^{\prime \mu \nu} F_{\mu \nu}^{\prime}\right)-\frac{M_{A^{\prime}}^{2}}{2} A_{\mu}^{\prime} A^{\prime \mu} \\
& -C_{V} \bar{f} \gamma^{\mu} f A_{\mu}^{\prime}+\ldots,
\end{aligned}
$$

where $M_{A^{\prime}}$ is the mass of the dark photon, $F_{\mu \nu}$ and $F_{\mu \nu}^{\prime}$ represent the field strength tensor of $A_{\mu}$ and $A_{\mu}^{\prime}$ respectively. $C_{V}$ is the vector couplings of the dark photon due to kinetic mixing with ordinary photons and $f$ is the Standard Model fermions. In this paper, we assume that the dark photon mass term $M_{A^{\prime}}$ is a Stueckelberg mass 38] instead of a Higgs mass. The Stueckelberg mass can be naturally induced from string compactifications, thus the dark photons can be safely regarded as massive without passing through a spontaneous symmetry breaking (SSB) phase transition until the very early universe. The misalignment mechanism then applies in the scenario. Interestingly, nature seems prefer to use every renormalizable Lagrangian term, yet the Stueckelberg mass is absent in the Standard Model. If the Stueckelberg mass exists, it applies only to abelian gauge fields as far as renormalizability is required, thus we may suspect 
that dark photons do exist in our universe.

The initial value of the $A_{\mu}^{\prime}$ field is assumed to be some random nonzero value, and the inflation creates a spatially uniform field, thus $\partial_{i} A_{\mu}^{\prime} \sim 0$. In a FriedmannRobertson-Walker (FRW) universe and the cosmic frame, the equation of motion of the vector field [31, 35] implies that $A_{0}^{\prime}=0$ if $M_{A^{\prime}} \neq 0$, and

$$
\begin{aligned}
\partial_{t}^{2} \vec{A}^{\prime}+3 H(t) \partial_{t} \vec{A}^{\prime} \\
+\left(M_{A^{\prime}}^{2}+\dot{H}(t)+2 H(t)^{2}\right) \vec{A}^{\prime}=0,
\end{aligned}
$$

where $H(t)$ is the Hubble parameter. After the universe enters a radiation dominated era Eq. 20 reduces to $\partial_{t}^{2} \vec{A}^{\prime}+(3 / 2 t) \partial_{t} \vec{A}^{\prime}+M_{A^{\prime}}^{2} \vec{A}^{\prime}=0$. The solution of the equation is $A_{i}^{\prime}=C_{1} J_{1 / 4}\left(M_{A^{\prime}} t\right) / t^{1 / 4}+C_{2} Y_{1 / 4}\left(M_{A^{\prime}} t\right) / t^{1 / 4}$, where $J_{N}, Y_{N}$ are the Bessel functions and $C_{1}, C_{2}$ depend on the initial conditions. The solution can be explained intuitively. When $H(t) \gg M_{A^{\prime}}$, the field strength is decreasing without any oscillations. Thus no particles are observed during this period, only a uniform field in the universe. After the Hubble constant decreases to a point that $3 H(t) \sim 2 M_{A^{\prime}}$, the field begins to oscillate. The amplitude of the initial oscillation determines the energy density of the new born particles so in this scenario we have a non-thermal creation mechanism. After $H \ll M_{A^{\prime}}$ the energy density of the vector field $\rho \sim A^{\prime 2}$ decreases as $1 / a^{3}(t)$ therefore behaves like dust. With a proper initial value and particle mass combination, the mechanism can provide the right energy density of the dark matter. As the transition happens at the same time in the universe, the particles are in a coherent state with small fluctuations and are very cold due to their coherent nature regardless of their mass.

The axion is introduced to provide a natural solution to the strong CP problem [39 44]. Generally speaking, to solve the strong $\mathrm{CP}$ problem, one may introduce a new $U(1)$ symmetry called the Peccei-Quinn symmetry. The Peccei-Quinn symmetry is spontaneously broken when the energy is below the scale $\Lambda \sim f_{a} \sim 10^{12} \mathrm{GeV}$ and subsequently is explicitly broken due to non-perturbative quantum chromodynamics (QCD) effects after the energy drops below the QCD scale. The SSB gives a goldstone boson and the explicitly symmetry breaking gives the goldstone boson a small non-zero mass. This pseudo-goldstone boson is now known as the axion. The axion-like particles [14, 17, 45 47] are zero Kaluza-Klein modes created from compactifying antisymmetric tensor fields on closed cycles of space-time manifold. These zero modes acquire a potential from non-perturbative effects on the cycle and therefore obtain a small mass. Although the compactifications give rise to many axionlike particles, we consider a scenario in which one of them composes the majority of dark matter. Both axions and axion-like particles have similar properties in low energy four-dimensional effective field theory, except in the case of axions the mass and the SSB scale are closely related. We can write the general Lagrangian for axions and axion-like particles as follows:

$\mathcal{L}=-\frac{1}{4} F^{\mu \nu} F_{\mu \nu}-\frac{1}{2} \partial_{\mu} \partial^{\mu} \phi-\frac{M_{\phi}^{2}}{2} \phi^{2}+\frac{\alpha g}{2 \pi \Lambda} \phi F_{\mu \nu} F_{\alpha \beta} \epsilon^{\mu \nu \alpha \beta}$

where $\Lambda$ is the order of symmetry breaking energy scale, $g$ is a model dependent factor of order one which we will neglect in the following discussion, $\alpha$ is the fine structure constant, and $\phi$ is the particle field. For the QCD axions we have [48]:

$$
M_{\phi} \sim m_{\pi^{0}} f_{\pi} / \Lambda \simeq 6.00 * 10^{-6} \mathrm{eV}\left(10^{12} \mathrm{GeV} / \Lambda\right) .
$$

In addition, inflation can produce isocurvature perturbations of scalar fields which are highly constrained from the observations of cosmic microwave background radiations. As such we have additional limitations for the axion [49 57].

Cold axion-like particles can be created from the misalignment mechanism [9 [12, 14]. The equation of motion of $\phi$ in a FRW universe is $\partial_{t}^{2} \phi+3 H \partial_{t} \phi+M_{\phi}^{2} \phi=0$, so the production mechanism is similar to that of the dark photon. In addition, the initial field value of axionlike particles is constrained as $\phi / \Lambda$ is corresponding to a phase value order of one. Let us use $t_{1} \sim 1 / M_{\phi}$ to denote the time that axion-like particle field starts to oscillate. The energy density of axion-like particles to the critical energy density during the matter dominated era is thus:

$$
\frac{\rho_{a}}{\rho_{c}} \sim \frac{1}{2} M_{\phi}^{2} \Lambda^{2}\left(\frac{a\left(t_{1}\right)}{a(t)}\right)^{3} / \rho_{c} \approx 3 \pi G \sqrt{M}_{\phi} \Lambda^{2} \sqrt{t}_{e}<1,
$$

where $a$ is the FRW scale factor and $t_{e}$ is the matterradiation equality time. so we have a lower boundary in Fig.4.

As the Compton wavelength of dark matter should be no longer than $1 \mathrm{kpc}$ to allow for the structure formation on the kpc scale [31], both dark photons and axionlike particles have a weak bound on the mass, which is $M>10^{-24} \mathrm{eV}$. In addition, the survival of dark matter requires particles to have lifetimes longer than the age of the universe. These constraints are shown in Fig.3 and Fig.4.

Decaying Signals from Dark Matter Halos: Sub-MeV massive dark photons are not completely stable because they can decay via channel $\gamma^{\prime} \rightarrow 3 \gamma$ due to the vector interaction. The axion-like particles can decay via $\phi \rightarrow$ $2 \gamma$. The decay rate for the dark photon is:

$$
\begin{aligned}
\Gamma_{A^{\prime}} & =2.725 \times 10^{-15} \frac{C_{V}^{2} M_{A^{\prime}}^{9}}{m_{e}^{8}} \\
& =0.895 * 10^{-45} \text { seconds }^{-1}\left(\frac{M_{A^{\prime}}}{\mathrm{eV}}\right)^{9} C_{V}^{2},
\end{aligned}
$$

where $m_{e}$ is the mass of electrons. The decay rate of the axion-like particle $\Gamma_{\phi}$ is:

$$
\begin{aligned}
\Gamma_{\phi} & =\frac{\alpha^{2}}{4 \pi^{3} \Lambda^{2}} M_{\phi}^{3} \\
& =6.53 * 10^{-10} \mathrm{~s}^{-1}\left(\frac{M_{\phi}}{\mathrm{eV}}\right)^{3}\left(\frac{\mathrm{GeV}}{\Lambda}\right)^{2} .
\end{aligned}
$$




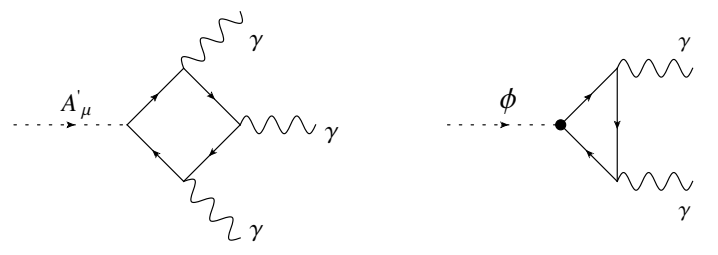

FIG. 1: The decay of a dark photon $A_{\mu}^{\prime}$ and of an axoin-like particle $\phi$.

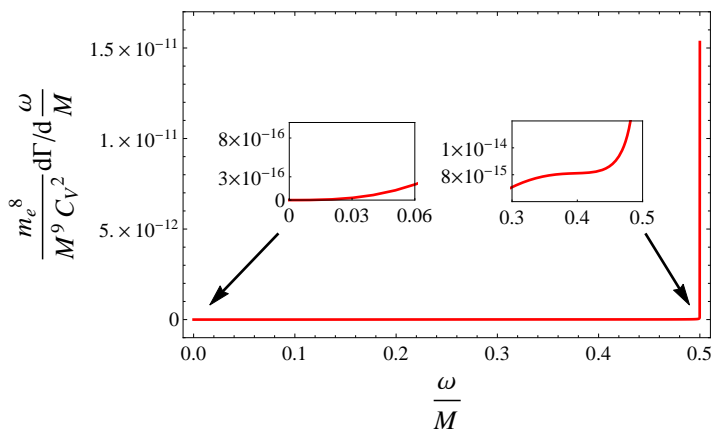

FIG. 2: The decay spectrum of the dark photon.

The axion-like particle decay is a two body decay and thus the resultant photons are monochromatic in the energy spectrum, with a frequency at $\omega=M / 2$. The dark photon decay is a three body decay, and the spectrum of the decaying photons is calculated and is shown in Fig.2. An interesting feature of the dark photon decay is that although it is a three body decay, which typically results to a smooth spectrum, e.g. in the $\beta$ decay, the decaying spectrum of the dark photon is however heavily peaked at $\omega=M / 2$. We find that $36.1 \%$ decaying energy lies in a frequency range $[0.9 * M / 2, M / 2]$.

The physical reason for the dark photon decay spectrum sharply peaking at $M / 2$ is due to the properties of the vector bosons and can be understood when we compare it with the orthopositronium decay spectrum. In the low energy end, both spectrums vanish linearly due to Low's theorem on soft photon emissions. In the $E \rightarrow M / 2$ end, both spectrums rise sharply, as the phase space for a two photon decay is far more allowed than that for a three photon decay. Therefore, in the three photon decay, one infrared photon plus two $M / 2$ photons is the most favorable situation. For the orthopositronium case, the phase space is much more tolerated as the initial state is different from a point particle. Therefore the spectrum of orthopositronium decay is much less peaked.

The spectrum of the decaying photons observed on Earth depends on several astronomical factors and there are four major sources that distort the spectrum: The first is the velocity dispersions $\delta v$ of the dark matter particles in a halo which lead to a $(1+\delta v / C) /(1-\delta v / C) \sim$ $2 \delta v / C$ spectrum spread. The second is the gravitational

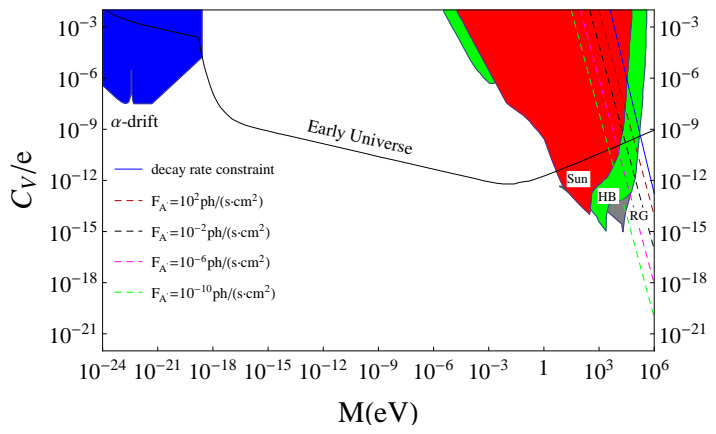

FIG. 3: The constraints and the equal flux lines of the sub$\mathrm{MeV}$ dark photon. The blue area is excluded from the drift of the fine structure constant and the early universe behavior imposes an upper limit for the coupling $C_{V}$ [31]. The red, green and gray areas are ruled out by the lifetime of stars (the sun, horizontal branch stars, red giants) [27, 32]. The lifetime of the dark photons impose a decay rate constraint for the upper bound of the mass. The structure formation imposes a lower bound of the mass $M_{A^{\prime}}>10^{-24} \mathrm{eV}$ 31]. The dashed lines are the equal light flux lines, showing that the photons are emitted from the M31 dark matter halo and received on earth.

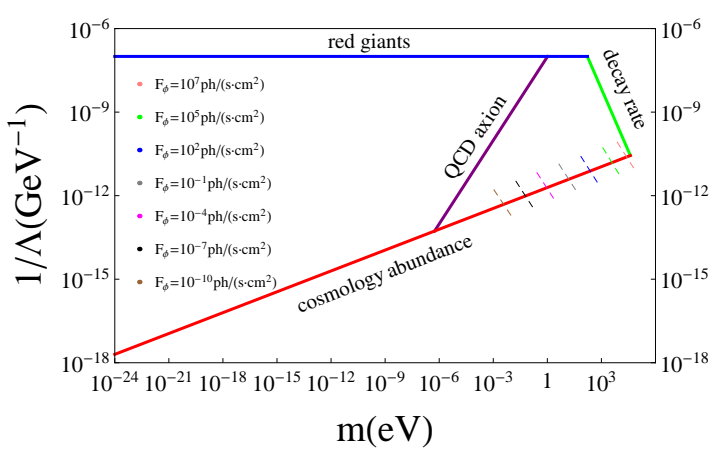

FIG. 4: The constraints and the equal flux lines of axionlike particles. The upper area is excluded from the stellar evolution constraint [33, 34]. The decay rate of the axion-like particle imposes an upper limit of the mass. The structure formation imposes a lower bound of the mass $M_{\phi}>10^{-24} \mathrm{eV}$. The lower area is excluded due to Eq.(15), as it would produce too much dark matter. For the QCD axion, the mass and the decay constant is related by Eq.(4). The dashed lines are the equal light flux lines showing that the photons are emitted from the M31 dark matter halo and received on earth. Note that for an axion-like particle to be a substantial fraction of dark matter, its parameters should lie around the cosmology abundance line.

red shifting due to local density profiles. The third is the kinetic movement of dark matter halos relative to the observer, and the fourth is the cosmological red shift due to expanse of the universe. Assuming the dark matter particles remain non-relativistic, $\delta v \ll 1$, the spectrum spread is small. Furthermore, the gravity induced spectrum spread should be small assuming a standard dark matter halo density profile. In addition, if the total mass 
of a source halo is similar to our galaxy halo, then the center frequency red shift due to photons moving in and out of halo gravity wells should be largely canceled. Finally, let us use $z_{k}$ and $z_{c}$ to denote the magnitude of the red shift due to movement of the halos and due to the cosmological red shift respectively. As $z_{k}$ and $z_{c}$ are small for nearby halos, the observed spectrum is peaked at $\left(1+z_{k}+z_{c}\right)^{-1} M / 2$.

To be a proper candidate for dark matter, the lifetime of particles $\tau=1 / \Gamma$ should be larger than the age of the universe. However, due to a huge particle number $N$ in a dark matter halo, the luminosity $L$ from decaying dark photons or decaying axion-like particles of a dark matter halo may be detectable. A galaxy halo is a heavy gravity bound structure with a typical mass ranging from $10^{11}$ to $10^{13}$ solar masses, and the most [1 7] of the halo mass is composed of dark matter. Let us consider a dark matter halo with a mass $M_{h}$. The luminosity of the dark photon halo is:

$$
\begin{aligned}
L_{A^{\prime}} & =\frac{N_{A^{\prime}} \times M}{\tau}=0.95 \frac{M_{h}}{\tau} \\
& =3.96 * 10^{-25} \frac{M_{h}}{M_{\odot}}\left(\frac{M}{\mathrm{eV}}\right)^{9} C_{V}^{2} L_{\odot} .
\end{aligned}
$$

For the axion-like particle dark matter halo the luminosity is:

$$
\begin{aligned}
L_{\phi} & =\frac{N_{\phi} \times M_{\phi}}{\tau} \\
& =28.9 * 10^{10} \frac{M_{h}}{M_{\odot}}\left(\frac{M_{\phi}}{\mathrm{eV}}\right)^{3}\left(\frac{\mathrm{GeV}}{\Lambda}\right)^{2} L_{\odot},
\end{aligned}
$$

where $M_{\odot}$ and $L_{\odot}$ denote the solar mass and the solar luminosity respectively. For QCD axions, the mass and the energy breaking scale are closely related by Eq.(4). As such we have $L_{\phi} \propto\left(M_{h} / M_{\odot}\right) *\left(M_{\phi} / \mathrm{eV}\right)^{5} L_{\odot}$ which is consistent with the literature [58 60].

The observed light flux at a distance is:

$$
F=\frac{L}{4 \pi d_{L}^{2}}
$$

where $d_{L}$ is the luminosity distance. For nearby galaxy halos it is sufficient to use $d_{L} \approx z_{c} c / H_{0}$, where $c$ is the speed of light. The Hubble constant $H_{0} \approx 73(\mathrm{~km} / \mathrm{s}) / \mathrm{Mpc} 61$, so we have $d_{L} \approx 4.11 *$ $10^{3} z_{c}$ Mpc. The observed flux within a frequency range $[0.9 M / 2, M / 2]$ from a dark photon galaxy halo with a cosmological red-shift $z_{c}$ is then:

$$
F_{A^{\prime}}=2.69 * 10^{-56} \frac{M_{h}}{M_{\odot}}\left(\frac{M}{\mathrm{eV}}\right)^{9} C_{V}^{2} z_{c}^{-2}\left(\mathrm{~W} / \mathrm{cm}^{2}\right)
$$

$$
=3.36 * 10^{-37} \frac{M_{h}}{M_{\odot}}\left(\frac{M}{\mathrm{eV}}\right)^{8} C_{V}^{2} z_{c}^{-2} \mathrm{ph} /\left(\mathrm{s} \mathrm{cm}^{2}\right)
$$

For the axion-like particle dark matter halo the observed flux is:

$$
\begin{aligned}
F_{\phi} & =5.46 * 10^{-20} \frac{M_{h}}{M_{\odot}}\left(\frac{M_{\phi}}{\mathrm{eV}}\right)^{3}\left(\frac{\mathrm{GeV}}{\Lambda}\right)^{2} z_{c}^{-2}\left(\mathrm{~W} / \mathrm{cm}^{2}\right) \\
& =0.683 * \frac{M_{h}}{M_{\odot}}\left(\frac{M_{\phi}}{\mathrm{eV}}\right)^{2}\left(\frac{\mathrm{GeV}}{\Lambda}\right)^{2} z_{c}^{-2} \mathrm{ph} /\left(\mathrm{s} \mathrm{cm}^{2}\right) .(1
\end{aligned}
$$

The NGC 224 or Messier 31 galaxy is a major neighbor galaxy near the earth. The distance from NGC224 to the earth is $0.778 \mathrm{Mpc}$ 62] which corresponds to a $z_{c}=1.89 * 10^{-4}$. The mass of NGC 224 is about $1.5 * 10^{12}$ 63 solar masses. Star formations in the NGC 224 disk are near inactive, and the galactic neutral hydrogen, molecular hydrogen and dust combined counts about $10^{10}$ solar masses or below $1 \%$ of the total masses 64]. Therefore the spectrum distortions should be small. We expect the received signal to be a narrow line that is peaked at $\left(1+z_{k}+z_{c}\right)^{-1} M / 2$ where $\left(z_{k}+z_{c}\right) \sim-10^{-3}$ for the NGC 224. If the dark matter is composed by the dark photon, then the luminosity of NGC 224 is $5.98 * 10^{6} L_{\odot}\left(\frac{M}{60 \mathrm{keV}}\right)^{9}\left(\frac{C_{V}}{10^{-12}}\right)^{2}$ and the flux within $[0.9 M / 2, M / 2]$ received on earth is $2.37 * 10^{-3}\left(\frac{M}{60 \mathrm{keV}}\right)^{8}\left(\frac{C_{V}}{10^{-12}}\right)^{2} \mathrm{ph} /\left(\mathrm{s} \mathrm{cm}^{2}\right)$. If the dark matter is composed of the axion/axion-like particles, then the luminosity and the flux are $0.434 L_{\odot}\left(\frac{M}{\mathrm{eV}}\right)^{3}\left(10^{12} \frac{\mathrm{GeV}}{\Lambda}\right)^{2}$ and $2.87 * 10^{-5}\left(\frac{M}{\mathrm{eV}}\right)^{2}\left(10^{12} \frac{\mathrm{GeV}}{\Lambda}\right)^{2} \mathrm{ph} /\left(\mathrm{s} \mathrm{cm}^{2}\right)$ respectively.

Conclusions: Boson condensate produced from the misalignment mechanism is a viable candidate for dark matter. The spin 0 axions/axion-like particles and the spin 1 dark photons are especially well motivated. The huge particle number in a galactic halo makes the decaying signal detectable for some regions of parameter space. Both types of dark matter candidate have a very sharp spectrum line peaked at $\left(1+z_{c}+z_{k}\right)^{-1} M / 2$. A scan of the relative spectrum of nearby galactic halos combined with complementary laboratory experiments may reveal the nature of dark matter.

Acknowledgments: Q. Yang would like to thank XiangSong Chen, JianWei Cui, WeiTian Deng, Bo Feng, YunGui Gong and YunQi Liu for their helpful discussions. This work is partially supported by the Natural Science Foundation of China under grant No. 11305066.
[1] G. Battaglia et al., "The Radial velocity dispersion profile of the Galactic Halo: Constraining the density profile of the dark halo of the
Milky Way," Mon. Not. Roy. Astron. Soc. 364, 433 (2005) Erratum: [Mon. Not. Roy. Astron. Soc. 370, 1055 (2006)] doi:10.1111/j.1365-2966.2006.10688.x, 
10.1111/j.1365-2966.2005.09367.x astro-ph/0506102.

[2] V. Springel et al., "Simulating the joint evolution of quasars, galaxies and their large-scale distribution," Nature 435, 629 (2005) doi:10.1038/nature03597 astro-ph/0504097.

[3] M. C. Smith et al., "The RAVE Survey: Constraining the Local Galactic Escape Speed," Mon. Not. Roy. Astron. Soc. 379, 755 (2007) doi:10.1111/j.13652966.2007.11964.x astro-ph/0611671.

[4] A. R. Duffy, J. Schaye, S. T. Kay and C. Dalla Vecchia, "Dark matter halo concentrations in the Wilkinson Microwave Anisotropy Probe year 5 cosmology," Mon. Not. Roy. Astron. Soc. 390, L64 (2008) Erratum: [Mon. Not. Roy. Astron. Soc. 415, L85 (2011)] doi:10.1111/j.17453933.2008.00537.x arXiv:0804.2486 [astro-ph]].

[5] N. Okabe, M. Takada, K. Umetsu, T. Futamase and G. P. Smith, "LoCuSS: Subaru Weak Lensing Study of 30 Galaxy Clusters," Publ. Astron. Soc. Jap. 62, 811 (2010) doi:10.1093/pasj/62.3.811 arXiv:0903.1103 [astro-ph.CO]].

[6] Q. Guo, S. White, C. Li and M. Boylan-Kolchin, "How do galaxies populate Dark Matter halos?," Mon. Not. Roy. Astron. Soc. 404, 1111 (2010) doi:10.1111/j.13652966.2010.16341.x arXiv:0909.4305 [astro-ph.CO]].

[7] M. Postman et al., "Cluster Lensing And Supernova survey with Hubble (CLASH): An Overview," Astrophys. J. Suppl. 199, 25 (2012) doi:10.1088/0067-0049/199/2/25 arXiv:1106.3328 [astro-ph.CO]].

[8] F. S. Queiroz, W. Rodejohann and C. E. Yaguna, "Is the dark matter particle its own antiparticle?," arXiv:1610.06581 [hep-ph].

[9] J. Ipser, P. Sikivie, "Are Galactic Halos Made Of Axions?," Phys. Rev. Lett. 50, 925 (1983).

[10] J. Preskill, M. B. Wise, F. Wilczek, "Cosmology of the Invisible Axion," Phys. Lett. B120, 127-132 (1983)

[11] L. F. Abbott, P. Sikivie, "A Cosmological Bound on the Invisible Axion," Phys. Lett. B120, 133-136 (1983).

[12] M. Dine, W. Fischler, "The Not So Harmless Axion," Phys. Lett. B120, 137-141 (1983).

[13] Z. G. Berezhiani, M. Y. Khlopov and R. R. Khomeriki, "Cosmic Nonthermal Electromagnetic Background from Axion Decays in the Models with Low Scale of Family Symmetry Breaking," Sov. J. Nucl. Phys. 52, 65 (1990) [Yad. Fiz. 52, 104 (1990)].

[14] P. Svrcek, E. Witten, "Axions In String Theory," JHEP 0606, 051 (2006). hep-th/0605206.

[15] A. De Angelis, M. Roncadelli and O. Mansutti, "Evidence for a new light spin-zero boson from cosmological gamma-ray propagation?," Phys. Rev. D 76, 121301 (2007) doi:10.1103/PhysRevD.76.121301 arXiv:0707.4312 [astro-ph]].

[16] M. Simet, D. Hooper and P. D. Serpico, "The Milky Way as a Kiloparsec-Scale Axionscope," Phys. Rev. D 77, 063001 (2008) doi:10.1103/PhysRevD.77.063001 arXiv:0712.2825 [astro-ph]].

[17] A. Arvanitaki, S. Dimopoulos, S. Dubovsky, N. Kaloper and J. March-Russell, "String Axiverse," Phys. Rev. D 81, 123530 (2010) arXiv:0905.4720 [hep-th]].

[18] J. Jaeckel and A. Ringwald, "The Low-Energy Frontier of Particle Physics," Ann. Rev. Nucl. Part. Sci. 60, 405 (2010) doi:10.1146/annurev.nucl.012809.104433 arXiv:1002.0329 [hep-ph]].

[19] A. De Angelis, G. Galanti and M. Roncadelli, "Relevance of axion-like particles for very-high-energy astrophysics," Phys. Rev. D 84, 105030 (2011) Erratum: [Phys. Rev. D 87, no. 10, 109903 (2013)] doi:10.1103/PhysRevD.87.109903, 10.1103/PhysRevD.84.105030 [arXiv:1106.1132 [astro-ph.HE]].

[20] A. Ringwald, "Exploring the Role of Axions and Other WISPs in the Dark Universe," Phys. Dark Univ. 1, 116 (2012) doi:10.1016/j.dark.2012.10.008 arXiv:1210.5081 [hep-ph]].

[21] D. J. E. Marsh, "Axion Cosmology," Phys. Rept. 643, 1 (2016) doi:10.1016/j.physrep.2016.06.005 arXiv:1510.07633 [astro-ph.CO]].

[22] S. A. Abel, M. D. Goodsell, J. Jaeckel, V. V. Khoze and A. Ringwald, "Kinetic Mixing of the Photon with Hidden U(1)s in String Phenomenology," JHEP 0807, 124 (2008) doi:10.1088/1126-6708/2008/07/124 arXiv:0803.1449 [hep-ph]].

[23] J. Redondo and M. Postma, "Massive hidden photons as lukewarm dark matter," JCAP 0902, 005 (2009) doi:10.1088/1475-7516/2009/02/005 arXiv:0811.0326 [hep-ph]].

[24] A. Mirizzi, J. Redondo and G. Sigl, "Microwave Background Constraints on Mixing of Photons with Hidden Photons," JCAP 0903, 026 (2009) doi:10.1088/14757516/2009/03/026 arXiv:0901.0014 [hep-ph]].

[25] M. Ahlers, H. Gies, J. Jaeckel, J. Redondo and A. Ringwald, "Light from the hidden sector," Phys. Rev. D 76, 115005 (2007) doi:10.1103/PhysRevD.76.115005 arXiv:0706.2836 [hep-ph]].

[26] J. Jaeckel, J. Redondo and A. Ringwald, "Signatures of a hidden cosmic microwave background," Phys. Rev. Lett. 101, 131801 (2008) doi:10.1103/PhysRevLett.101.131801 [arXiv:0804.4157 [astro-ph]].

[27] M. Goodsell, J. Jaeckel, J. Redondo and A. Ringwald, "Naturally Light Hidden Photons in LARGE Volume String Compactifications," JHEP 0911, 027 (2009) doi:10.1088/1126-6708/2009/11/027 arXiv:0909.0515 [hep-ph]].

[28] M. Bullimore, J. P. Conlon and L. T. Witkowski, "Kinetic mixing of U(1)s for local string models," JHEP 1011, 142 (2010) doi:10.1007/JHEP11(2010)142 arXiv:1009.2380 [hep-th]].

[29] J. Redondo and G. Raffelt, "Solar constraints on hidden photons re-visited," JCAP 1308, 034 (2013) doi:10.1088/1475-7516/2013/08/034 arXiv:1305.2920 [hep-ph]].

[30] H. An, M. Pospelov and J. Pradler, "New stellar constraints on dark photons," Phys. Lett. B 725, 190 (2013) doi:10.1016/j.physletb.2013.07.008 arXiv:1302.3884 [hep-ph]].

[31] A. E. Nelson and J. Scholtz, "Dark Light, Dark Matter and the Misalignment Mechanism," Phys. Rev. D 84, 103501 (2011) doi:10.1103/PhysRevD.84.103501 arXiv:1105.2812 [hep-ph]].

[32] I. M. Bloch, R. Essig, K. Tobioka, T. Volansky and T. T. Yu, "Searching for Dark Absorption with Direct Detection Experiments," arXiv:1608.02123 [hep-ph].

[33] A. Ayala, I. Domnguez, M. Giannotti, A. Mirizzi and O. Straniero, "Revisiting the bound on axion-photon coupling from Globular Clusters," Phys. Rev. Lett. 113, no. 19, 191302 (2014) doi:10.1103/PhysRevLett.113.191302 arXiv:1406.6053 [astro-ph.SR]].

[34] M. Giannotti, I. Irastorza, J. Redondo and A. Ringwald, "Cool WISPs for stellar cooling excesses," JCAP 1605, no. 05, 057 (2016) doi:10.1088/1475-7516/2016/05/057 
arXiv:1512.08108 [astro-ph.HE]].

[35] P. Arias, D. Cadamuro, M. Goodsell, J. Jaeckel, J. Redondo and A. Ringwald, "WISPy Cold Dark Matter," JCAP 1206, 013 (2012) doi:10.1088/14757516/2012/06/013 arXiv:1201.5902 [hep-ph]].

[36] P. Sikivie and Q. Yang, "Bose-Einstein Condensation of Dark Matter Axions," Phys. Rev. Lett. 103, 111301 (2009) doi:10.1103/PhysRevLett.103.111301 arXiv:0901.1106 [hep-ph]].

[37] A. V. Manohar and P. Ruiz-Femenia, "The Orthopositronium decay spectrum using NRQED," Phys. Rev. D 69, 053003 (2004) doi:10.1103/PhysRevD.69.053003 hep-ph/0311002.

[38] D. Feldman, Z. Liu and P. Nath, "The Stueckelberg Zprime Extension with Kinetic Mixing and Milli-Charged Dark Matter From the Hidden Sector," Phys. Rev. D 75, 115001 (2007) doi:10.1103/PhysRevD.75.115001 hep-ph/0702123 [HEP-PH]].

[39] R. D. Peccei, H. R. Quinn, "CP Conservation in the Presence of Instantons," Phys. Rev. Lett. 38, 1440-1443 (1977).

[40] S. Weinberg, "A New Light Boson?," Phys. Rev. Lett. 40, 223 (1978).

[41] F. Wilczek, "Problem of Strong P and T Invariance in the Presence of Instantons," Phys. Rev. Lett. 40, 279 (1978).

[42] J. E. Kim, "Weak Interaction Singlet and Strong CP Invariance," Phys. Rev. Lett. 43, 103 (1979).

[43] M. A. Shifman, A. I. Vainshtein, V. I. Zakharov, "Can Confinement Ensure Natural CP Invariance of Strong Interactions?," Nucl. Phys. B166, 493 (1980).

[44] M. Dine, W. Fischler, M. Srednicki, "A Simple Solution to the Strong CP Problem with a Harmless Axion," Phys. Lett. B104, 199 (1981).

[45] K. Becker, M. Becker and A. Strominger, "Five-branes, membranes and nonperturbative string theory," Nucl. Phys. B 456, 130 (1995) hep-th/9507158.

[46] R. Kallosh, A. D. Linde, D. A. Linde and L. Susskind, "Gravity and global symmetries," Phys. Rev. D 52, 912 (1995) hep-th/9502069.

[47] M. R. Douglas and S. Kachru, "Flux compactification," Rev. Mod. Phys. 79, 733 (2007) hep-th/0610102.

[48] P. Sikivie, "Axion Cosmology," Lect. Notes Phys. 741, 19 (2008) astro-ph/0610440.

[49] M. S. Turner, "Cosmic and Local Mass Density of Invisible Axions," Phys. Rev. D 33, 889 (1986). doi:10.1103/PhysRevD.33.889

[50] D. H. Lyth, "A Limit on the Inflationary Energy Density From Axion Isocurvature Fluctuations," Phys. Lett. B 236, 408 (1990). doi:10.1016/0370-2693(90)90374-F

[51] M. S. Turner and F. Wilczek, "Inflationary ax- ion cosmology," Phys. Rev. Lett. 66, 5 (1991). doi:10.1103/PhysRevLett.66.5

[52] D. H. Lyth and E. D. Stewart, "Constraining the inflationary energy scale from axion cosmology," Phys. Lett. B 283, 189 (1992). doi:10.1016/0370-2693(92)90006-P

[53] S. D. Burns, "Isentropic and isocurvature axion perturbations in inflationary cosmology," astro-ph/9711303

[54] P. Fox, A. Pierce and S. D. Thomas, "Probing a QCD string axion with precision cosmological measurements," hep-th/0409059.

[55] M. Beltran, J. Garcia-Bellido and J. Lesgourgues, "Isocurvature bounds on axions revisited," Phys. Rev. D 75, 103507 (2007) doi:10.1103/PhysRevD.75.103507 hep-ph/0606107.

[56] M. P. Hertzberg, M. Tegmark and F. Wilczek, "Axion Cosmology and the Energy Scale of Inflation," Phys. Rev. D 78, 083507 (2008) doi:10.1103/PhysRevD.78.083507 arXiv:0807.1726 [astro-ph]].

[57] M. Estevez and O. Santillan, "About the isocurvature tension between axion and high scale inflationary models," Eur. Phys. J. C 76, no. 7, 398 (2016) doi:10.1140/epjc/s10052-016-4226-2 arXiv:1606.02389 [hep-ph]].

[58] T. W. Kephart and T. J. Weiler, "Luminous Axion Clusters," Phys. Rev. Lett. 58, 171 (1987). doi:10.1103/PhysRevLett.58.171

[59] M. A. Bershady, M. T. Ressell and M. S. Turner, "Telescope search for multi-eV axions," Phys. Rev. Lett. 66, 1398 (1991). doi:10.1103/PhysRevLett.66.1398

[60] D. Grin, G. Covone, J. P. Kneib, M. Kamionkowski, A. Blain and E. Jullo, "A Telescope Search for Decaying Relic Axions," Phys. Rev. D 75, 105018 (2007) doi:10.1103/PhysRevD.75.105018 astro-ph/0611502.

[61] A. G. Riess et al., "A $2.4 \%$ Determination of the Local Value of the Hubble Constant," Astrophys. J. 826, no. 1, 56 (2016) doi:10.3847/0004-637X/826/1/56 arXiv:1604.01424 [astro-ph.CO]].

[62] I. Ribas, C. Jordi, F. Vilardell, E. L. Fitzpatrick, R. W. Hilditch and E. F. Guinan, "First determination of the distance and fundamental properties of an eclipsing binary in the andromeda galaxy," Astrophys. J. 635, L37 (2005) doi:10.1086/499161 astro-ph/0511045.

[63] J. Penarrubia, Y. Z. Ma, M. G. Walker and A. McConnachie, "A dynamical model of the local cosmic expansion," Mon. Not. Roy. Astron. Soc. 443, no. 3, 2204 (2014) doi:10.1093/mnras/stu879 arXiv:1405.0306 [astro-ph.GA]].

[64] B. T. Draine et al., "Andromeda's Dust," Astrophys. J. 780, 172 (2014) doi:10.1088/0004-637X/780/2/172 arXiv:1306.2304 [astro-ph.CO]]. 